\title{
NUEVO REGISTRO DE BLECHNUM (BLECHNACEAE) PARA LA FLORA DEL NOROESTE ARGENTINO
}

\author{
Adrián M. Jarsun ${ }^{1}$, Cristina J. Chambi² \& Olga G. Martínez ${ }^{1,2}$ \\ ${ }^{1}$ Instituto de Bio y Geociencias del Noroeste Argentino (IBIGEO-CONICET), Universidad Nacional de Salta, Av. \\ Bolivia 5150, 4400 Salta, Argentina; adrianjarsun@gmail.com (autor corresponsal). \\ ${ }^{2}$ Herbario MCNS, Facultad de Ciencias Naturales, Universidad Nacional de Salta, Av. Bolivia 5150, 4400 Salta, Argentina.
}

\begin{abstract}
Jarsun A. M.; C. J. Chambi \& O. G. Martínez. 2018. New record of Blechnum (Blechnaceae) for the Flora of northwestern Argentina. Darwiniana, nueva serie 6(2): 191-195.

Blechnum malacothrix, until now known from Peru and Bolivia, is registered for the first time in northwestern Argentina. With this finding the number of species of the Blechnaceae in this country ascends to 23, most of them growing in the north. A description of Blechnum malacothrix, together with illustrations of the specie, a map with the new collection site, and a key to identify the species of Blechnum growing in northwestern Argentina are presented.
\end{abstract}

Keywords. Blechnum; ferns diversity; monomorphic fronds; northwestern Argentina.

Resumen. Jarsun A. M.; C. J. Chambi \& O. G. Martínez. 2018. Nuevo registro de Blechnum (Blechnaceae) para la flora del noroeste argentino. Darwiniana, nueva serie 6(2): 191-195.

Blechnum malacothrix, hasta el momento conocida sólo de Perú y Bolivia, se registra por primera vez para el noroeste de Argentina. Con este hallazgo el número de especies de la familia Blechnaceae en este país asciende a 23, la mayoría de las cuales crece en el norte. Se presenta una descripción de Blechnum malacothrix, junto con ilustraciones, un mapa del nuevo sitio de colección y una clave para identificar las especies de Blechnum que crecen en el noroeste argentino.

Palabras clave. Blechnum, diversidad de helechos; frondes monomórficas; noroeste argentino.

\section{INTRODUCCIÓN}

La familia Blechnaceae Newman incluye entre 200 y 267 especies reunidas en tres subfamilias y 24 géneros (Gasper et al., 2016). Su distribución es subcosmopolita, con mayor número de representantes en el hemisferio sur (Kramer et al., 1990), donde se encuentran dos centros de diversificación: el Neotrópico y Oceanía/ Australasia (Dittrich et al., 2007).

El género Blechnum L., recientemente circunscripto (Gasper et al., 2017), comprende ca. 30 especies, la mayoría neotropicales, con dos especies africanas (Gasper et al., 2017). Son plantas predominantemente terrestres, saxícolas a hemiepífitas, con rizomas erectos a ascendentes, decumbentes o rastreros, estoloníferos, delgados a algo robustos; frondes generalmente monomórficas, en algunos casos subdimórficas, con láminas pinnatífidas a pinnadas, raras veces enteras, pinnas sésiles, adnatas o subpecioluladas, lanceoladas, oblongo-lanceoladas, con el margen ligeramente denticulado y venación libre o raro parcialmente areolada (Tryon \& Tryon, 1982; Gasper et al., 2016). 
Para el noroeste argentino, de la Sota (1977), Rolleri \& Prada (2006) y Ramos Giacosa (2016) citaron 11 especies de Blechnum en sentido amplio. Según la actual clasificación filogenética de Gasper et al. (2017), estas especies están comprendidas en seis géneros y se enumeran a continuación: Austroblechnum lechleri (T. Moore) Gasper \& V.A.O. Dittrich, A. penna-marina (Poir.) Gasper \& V.A.O. Dittrich, A. squamipes (Hieron.) Gasper \& V.A.O. Dittrich; Blechnum laevigatum Cav., $B$. auriculatum Cav., B. austrobrasilianum de la Sota, B. occidentale L.; Cranfillia sprucei (C. Chr.) Gasper \& V.A.O. Dittrich; Lomariocycas yungensis (Ramos Giacosa) Gasper \& A.R. Sm.: Neoblechnum brasiliense (Desv.) Gasper \& V.A.O. Dittrich y Parablechnum cordatum (Desv.) Gasper \& Salino.

Recientemente, en el marco de los estudios de los helechos de la provincia de Salta, se ha identificado a Blechnum malacothrix Maxon \& C.V. Morton, un nuevo hallazgo para la flora del noroeste argentino, hasta ahora conocido para Bolivia y Perú (Jörgensen et al., 2014; Rolleri \& Prada, 2006; Smith \& Kessler, 2018).

\section{MATERIALES Y MÉTODOS}

El material estudiado fue colectado en campañas realizadas en el Departamento Orán, en la provincia de Salta. El mismo se encuentra depositado en los herbarios MCNS y SI (Thiers, en constante actualización). La identificación taxonómica se realizó según el método tipológico, consultando los tipos nomenclaturales y mediante bibliografía específica: Maxon \& Morton (1939), Kessler \& Smith (2017) y Smith \& Kessler (2018). El material tipo se analizó a través de las imágenes digitales disponibles en Jstor Plant Science (http://plants.jstor.org).

Los caracteres diagnósticos considerados en la identificación del taxón fueron: forma y tamaño de las frondes, morfología de las escamas e indumento.

Las observaciones y fotografías se realizaron con microscopios Zeiss Primo Star y DV 4.

\section{RESULTADOS}

Blechnum malacothrix Maxon \& C.V. Morton. Bull. Torrey Bot. Club 66 (1): 40. 1939. TIPO: Bolivia, Okara, Cordillera Real, 2250 m s.m., 29-IV-1926, G. H. H. Tate 951 (holotipo NY 00149767!, isotipo US 00067432 !). Figs. 1 y 2.

Plantas terrestres con rizoma cortamente rastrero, de 1,5-2 $\mathrm{mm}$ de diámetro; escamas ovadas, con base cordada, ápice atenuado, margen con escasos pelos, de 0,5-1 $\times$ 0,5$0,55 \mathrm{~mm}$ de largo, brillantes, con área media esclerosada y márgenes hialinos. Frondes monomorfas de 10-21(-25) × 1-3,5(-4) cm, frondes fértiles levemente más largas y más angostas que las estériles. Peciolos de $2-8 \mathrm{~cm}$ de largo, escamosos en la base, con escamas deltoideas translúcidas con centro esclerosado, de 1-1,2 × 0,5-0,7 mm, márgenes denticulados; eje surcado adaxialmente, estramíneo, glandularpiloso, con pelos 3-5 celulares, translúcidos, caedizos. Láminas linear-lanceoladas, pinnadas a pinnado-pinnatisectas, base truncada a levemente reducida, ápice subcaudado, membranáceas, glandular-pilosas sobre ambos lados. Pinnas hasta 15-21(-25) pares, pinnas fértiles de $13-17 \times 3-5 \mathrm{~mm}$, pinnas estériles de $14-18 \times 4-5(-6) \mathrm{mm}$, algo distanciadas en la base, subascendentes, opuestas a subopuestas, rectas a falcadas, adnatas en la base, con escasos pelos translucidos, levemente auriculadas hacia lado acroscópico, ápice obtuso, márgenes suavemente cartilaginosos, con pequeños dentículos cristalinos; venación abierta 1-2-furcada. Raquis surcado adaxialmente, glandular-piloso, con pelos translúcidos. Cenosoros costales, de 2-8(-12) $\mathrm{mm}$ de largo, con indusios glabros, de 0,5-0,7 $\mathrm{mm}$ de ancho, con margen irregular. Esporangios con 64 esporas. Esporas monoletes, amarillentas, con perisporio liso y diámetro ecuatorial de 26(33)51 $\mu \mathrm{m}$.

Distribución geográfica y hábitat. En Argentina, Blechnum malacothrix crece en el sotobosque del piso de Mirtáceas, en la Selva Montana de las Yungas, por arriba de los 1000 m s.m. (Fig. 3). 


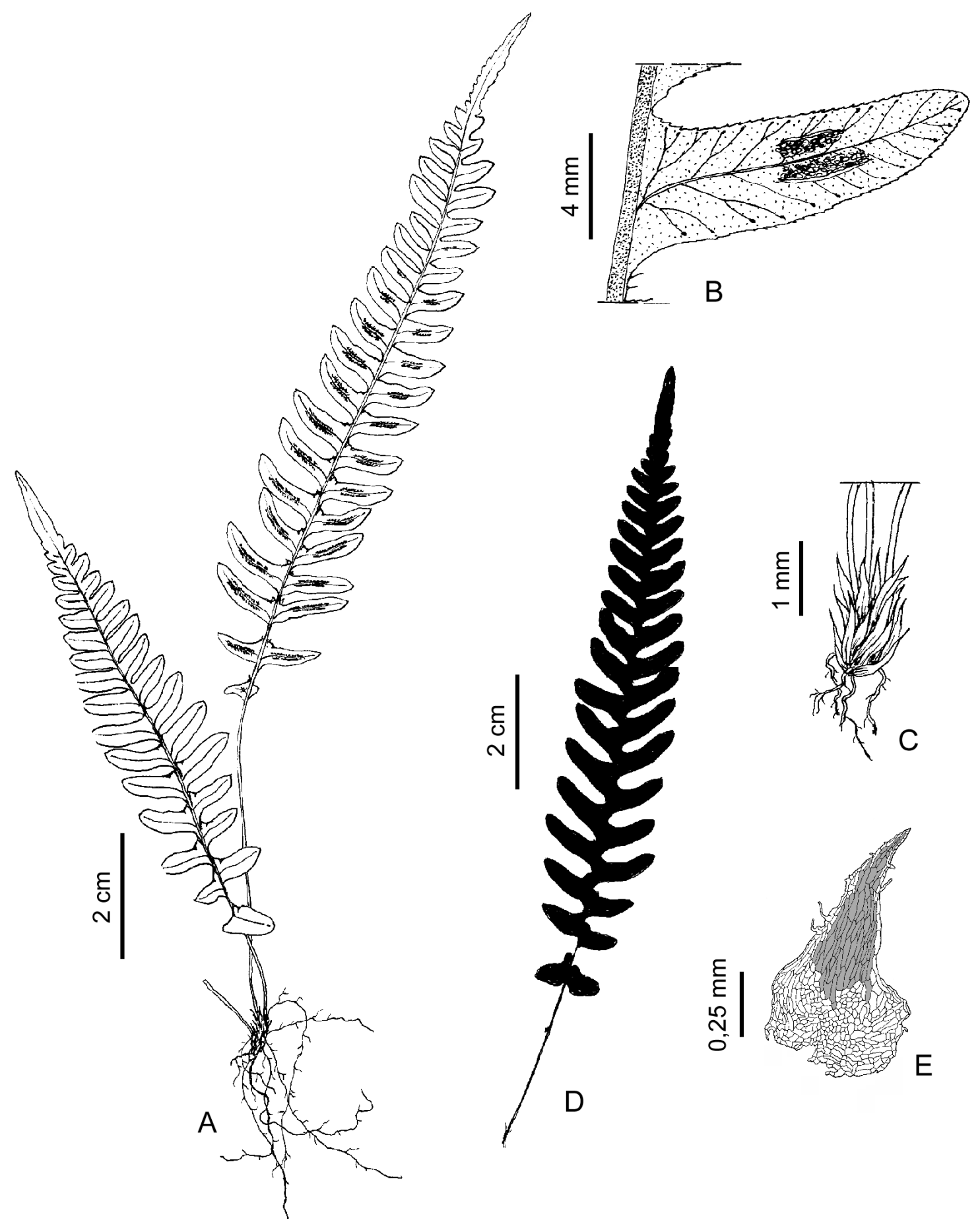

Fig. 1. Blechnum malacothrix. A, aspecto de la planta. B, detalle de pinna en cara abaxial. C, base de los pecíolos con escamas. D, silueta de fronde. E, escama rizomática. De Jarsun et al. 138 (MCNS). 

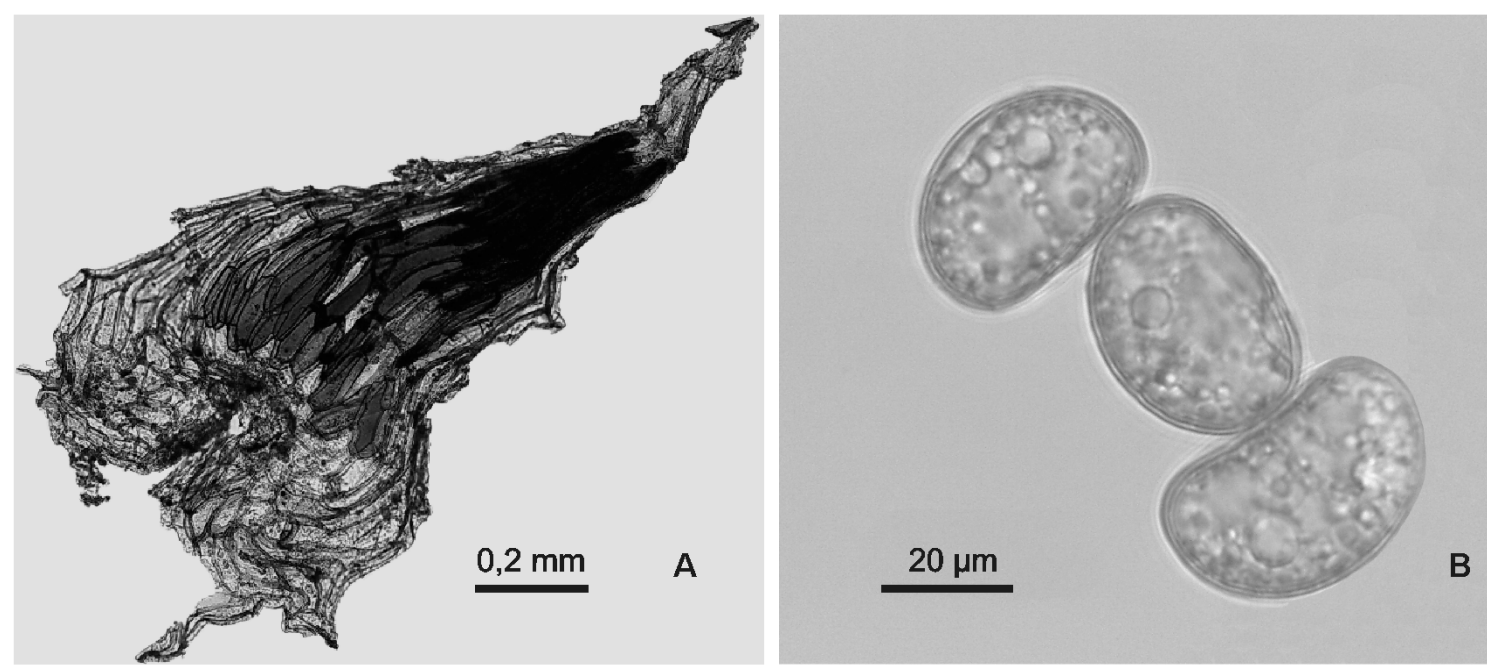

Fig. 2. Fotografías de Blechnum malacothrix: A, escama rizomática. B, esporas en vista ecuatorial.

En Bolivia se encuentra en los departamentos de Cochabamba, Chuquisaca, La Paz y Santa Cruz, entre los 650 y 2350 m s.m. (Jörgensen et al., 2014; Rolleri \& Prada, 2006; Smith \& Kessler, 2018).

\section{Material representativo examinado}

ARGENTINA. Salta. Depto. Orán, Angosto de Paraní, 1077 m s.m., 25-XI-2015, Jarsun et al. 138 (MCNS, SI); Angosto de Paraní, 1070 m s.m., 27-XI-2015, Chambi et al. 694 (MCNS).

\section{DISCUSIÓN Y CONCLUSIÓN}

Con este nuevo registro, las Blechnaceae suman a un total de 23 especies en la Argentina, agrupadas en dos subfamilias, Blechnoideae Gasper, V.A.O. Dittrich \& Salino y Stenochlaenoideae Roux emend. Gasper, V.A.O. Dittrich \& Salino, y en ocho géneros: Austroblechnum Gasper \& V.A.O. Dittrich, Blechnum L., Cranfillia Gasper \& V.A.O. Dittrich, Lomaridium C. Presl, Lomariocycas (J. Sm.) Gasper \& A.R. Sm., Neoblechnum Gasper \& V.A.O. Dittrich, Parablechnum C. Presl y Telmatoblechnum Perrie, D.J. Ohlsen \& Brownsey.
En el noroeste argentino las especies de Blechnaceae crecen principalmente en la provincia fitogeográfica de las Yungas, en la Selva y el Bosque Montano (Cabrera \& Willink, 1980), región correspondiente a los llamados "Bosques nublados" (Brown et al., 2001). El género mejor representado en esta región es Blechnum, con cinco especies. Blechnum malacothrix habita en la Selva Montana, distrito de las Yungas que se extiende entre los 700 y 1600 m s.m., y es probable su presencia en provincias vecinas que comparten este tipo de vegetación.

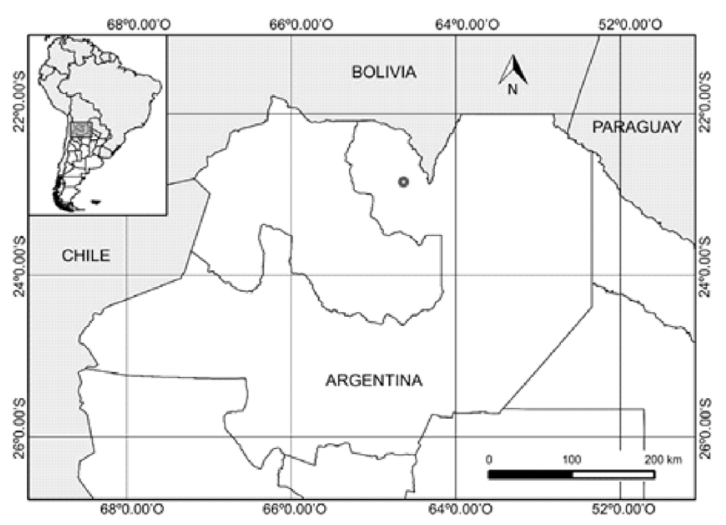

Fig. 3. Sitio de colección de Blechnum malacothrix en el noroeste argentino. 


\section{Clave para identificar las especies del género Blechnum que crecen en el noroeste argentino}

1. Frondes subdimórficas, láminas linear-lanceoladas, con la base atenuada; cenosoros entre la costa y el margen .......

B. auriculatum

1. Frondes monomórficas, láminas triangular lanceoladas, elíptico o linear-lanceoladas, con la base truncada o atenuada, cenosoros costales o marginales 2

2(1). Plantas mayores de $35 \mathrm{~cm}$ de largo; láminas papiráceas a subcoriáceas con márgenes y superficie glabra, con pelos sobre el raquis y parte basal de las costas en la cara abaxial 3

2. Plantas menores de $35 \mathrm{~cm}$ de largo; láminas membranáceas con escasos pelos translúcidos en el márgen; superficie y ejes glandular-pubescentes

4

3(2). Láminas papiráceas; pinnas lineares próximas entre sí; pinnas basales no reducidas, sésiles, margen acroscópico adnato y basiscópico con aurícula

B. austrobrasilianum

3. Láminas subcoriáceas; pinnas falcadas algo distanciadas entre sí; pinnas basales levemente reducidas, sésiles a brevemente pecioluladas, con la base redondeada a auriculada (a veces con lóbulos sobrepuestos al raquis)

B. occidentale

4(2). Frondes de 8,5 a $35 \mathrm{~cm}$ de largo; escamas rizomáticas castañas, concoloras, de 1,5 a 2,5 mm de longitud; láminas ovado-lanceoladas; pinnas de 5 a $10 \mathrm{~mm}$ de ancho

B. laevigatum

4. Frondes de 10 a $25 \mathrm{~cm}$ de largo; escamas rizomáticas con el centro esclerosado y margen translúcido, de $0,5 \mathrm{a} 1 \mathrm{~mm}$ de longitud; láminas linear-lanceoladas; pinnas de 3 a 5 (-6) $\mathrm{mm}$ de ancho B. malacothrix

\section{AGRADECIMIENTOS}

Agradecemos a María del C. Otero por las ilustraciones y al Consejo de Investigación de la Universidad Nacional de Salta (Proy. A 2344/0) por el financiamiento para este estudio.

\section{BIBLIOGRÁFÍA}

Brown, A. D.; H. R. Grau; L. R. Malizia \& A. Grau. 2001. Bosques Nublados del Neotrópico: Argentina. En Kapelle M. \& A. D. Brown (eds.), Bosques Nublados del Neotrópico, Ed. INBIO, Costa Rica, pp. 623-659.

Cabrera, A. L. \& A. Willink. 1980. Biogeografía de América Latina, $2^{\text {a }}$ Ed. Serie de Biología, Monografia 13. Washington D.C.: Secretaría General de la OEA (Publ. orig. 1973).

de la Sota, E. R. 1977. Pteridophyta. En Cabrera A. L. (Ed.). Flora de la Provincia de Jujuy. Colección Cientifica del Instituto Nacional de Tecnología Agropecuaria, 13(8): $1-275$.

Dittrich, V. A. O.; G. Heringer \& A. Salino. 2007. Blechnaceae. En: Cavalcanti, T. B.; Ramos, A. E. (Org.), Flora do Distrito Federal, Brasil 6: 91-108. Brasília: Embrapa Recursos Genéticos e Biotecnologia.

Gasper, A. L. de; V. A. O. Dittrich; A. R. Smith \& A. Salino. 2016. A classification for Blechnaceae (PolypodialesPloypodiopsida): New genera, resurrected names and combinations. Phytotaxa, 275 (3): 191-227.

Gasper, A. L. de; T. E. Almeida; V. A. O. Dittrich; A. R. Smith \& A. Salino. 2017. Molecular phylogeny of the fern family
Blechnaceae (Polypodiales) with a revised genus-level treatment. Cladistics 33 (4): 429-446.

Jörgensen, P. M.; M. Nee \& S. Beck. 2014. Catálogo de las Plantas Vasculares de Bolivia. Monographs in Systematic Botany from the Missouri Botanical Garden 127 (1-2): i-viii, 1-1744.

Kessler, M. \& A. R. Smith. 2017. Prodromus of a Fern Flora for Bolivia. I. General Introduction and Key to Families. Phytotaxa 327 (1): 57-89.

Kramer, K. U.; T. C. Chambers \& E. Hennipman. 1990. Blechnaceae. En Kramer, K. U. \& P. S. Green (eds.), The Families and Genera of Vascular Plants 1: 60-68. Pteridophytes and Gymnosperms. Springer-Verlag, Berlín.

Maxon, W. R. \& C. V. Morton. 1939. New Ferns from Bolivia and Peru. Bulletin of the Torrey Botanical Club 66 (1): 39-45.

Ramos Giacosa, J. P. 2016. Blechnaceae. En Ponce, M. M.; Arana, M. D. (coords.), Zuloaga, F. O. \& Belgrano, M. J. (eds.). Flora Argentina 2 (Licófitas, Helechos y Gymnospermae): 86-104.

Rolleri, C. H. \& C. Prada. 2006. Catálogo comentado de las especies de Blechnum L. (Blechnaceae-Pteridophyta) de Mesoamérica y Sudamérica. Anales del Jardín Botánico de Madrid 63 (1): 67-106.

Smith, A. R. \& M. Kessler. 2018. Prodromus of a fern flora for Bolivia. XXXIII. Blechnaceae. Phytotaxa 334 (2): 99-117.

Thiers, B. (permanentemente actualizado, 2018). Index Herbariorum: A global directory of public herbaria and associated staff. New York Botanical Garden's Virtual Herbarium. http://sweetgum.nybg.org/science/ih/ (consultado en marzo 2018).

Tryon, R. M. \& A. F. Tryon. 1982. Blechnacea: 662-683. Ferns and allied plants with special reference to Tropical America. Springer-Verlag, New York. 